Gómez Gómez, M. \& García Aretio, L. (2016). La formación como factor clave en la integración de la Pizarra Digital Interactiva. Perspectivas de profesores y coordinadores TIC. Revista Electrónica Interuniversitaria de Formación del Profesorado, 19 (3), 35-51.

\title{
La formación como factor clave en la integración de la Pizarra Digital Interactiva. Perspectivas de profesores y coordinadores TIC.
}

\author{
Marta Gómez Gómez ${ }^{(1)}$, Lorenzo García Aretio ${ }^{(2)}$ \\ Universidad Rey Juan Carlos ${ }^{(1)}$, Universidad Nacional de Educación a Distancia (2)
}

\section{Resumen}

En las dos últimas décadas se han ido percibiendo cada vez más centros educativos interesados en contar con las tecnologías en sus aulas. En esta situación se encuentran los colegios públicos de Educación Infantil y Primaria de Alcorcón, Madrid. Durante el curso 2010/2011 el ayuntamiento de este municipio implantó 400 pizarras digitales interactivas en dichos centros. El primer objetivo del estudio es conocer su impacto inicial en el proceso de enseñanza-aprendizaje a través de la percepción de profesores encuestados, como principales agentes de cambio. En el curso 2013/2014, trascurridos varios años, se pretendió conocer la evolución del recurso, para lo cual se entrevistaron a sus coordinadores TIC. La metodología empleada ha sido mixta y los principales resultados demuestran una buena acogida inicial a pesar de diferentes inconvenientes encontrados, tales como la escasa formación recibida, problemas técnicos, gran inversión de tiempo, etc. En definitiva, se puede concluir que la motivación, el cambio en la metodología y en la evaluación y, sobre todo, una adecuada formación técnico-pedagógica son claves para su exitosa integración.

\section{Palabras clave}

Tecnologías educativas; pizarras digitales interactivas; competencias; interacción.

\section{Contacto:}

Marta Gómez Gómez, marta.gomez@urjc.es, Universidad Rey Juan Carlos, C/ Tulipán s/n. Despacho 003, Laboratorio III. Campus de Móstoles. CP 28933.

La investigación realizada es una colaboración con la Concejalía de Educación y Deportes de Alcorcón en el Proyecto municipal de nuevas tecnologías en colegios públicos, y enmarcada en el proyecto “Innova, Alcorcón” (TSI-05100-2009-42). 


\title{
Teacher training as a key factor in the integration of Digital Interactive Whiteboards. Perspectives of teachers and ICT coordinators.
}

\begin{abstract}
In the last two decades, there has been an increasing number of educational institutions interested in adopting ICTs in their classrooms. This is the situation of early childhood and primary education state schools in the city of Alcorcón, Madrid. During the 2010/2011 school year, the government of this city council implanted 400 digital interactive whiteboards in these schools. The first objective is to know the initial impact on the teaching and learning process, through the teachers surveyed, as the main agents of change. In 2013/2014 school year, after several years, it is intended to know the evolution of the resource through the ICT coordinators interviewed. A mixed methodology has been employed and the main results demonstrated a good initial adoption, despite different drawbacks, such as the poor training received, technical problems, large investment of time, etc. Definitely, it can be concluded that the motivation, the change in methodology and assessment and, especially, the technical and pedagogical training are key to successful integration.
\end{abstract}

\section{Key words}

Educational technologies; digital interactive whiteboards; skills; interaction.

\section{Introducción}

En los últimos años, en todos los ámbitos de nuestra sociedad se han experimentado cambios que precisan de una nueva visión, misión y nuevas responsabilidades para adaptarnos a ellos eficazmente. Entre ellos, el uso continuado de todo tipo de tecnologías ha supuesto un mayor acceso a la información, diferentes maneras de comunicarnos, de trabajar y también nuevas formas de enseñar y aprender (OCDE, 2013). Es por ello por lo que cada vez surge un mayor interés en el estudio de los nuevos recursos y herramientas de la educación de hoy y su efecto en el desarrollo de nuestros alumnos.

Un factor importante es el esfuerzo económico y político que se está realizando en relación a la universalización de las tecnologías educativas, destacando iniciativas en Italia (Campregher, 2010), Latinoamérica (Gustavo, 2014), Reino Unido y Estados Unidos (Di Gregorio y Sobel-Lojeski, 2010), Australia (Lan y Hsiao, 2010), en Chile con el proyecto Enlaces (Gatica y Valdivia, 2013), el proyecto BECTA en Reino Unido (BECTA, 2004), etc.

En el contexto educativo, las tecnologías de la información y la comunicación (TICS) ya no son una novedad, sino una realidad y cada vez surgen más iniciativas al respecto. En el caso de nuestro país, al estar las competencias en materia de educación transferidas a las comunidades autónomas estos proyectos han sido muy diferentes entre sí. En este sentido, 
Area (2006) repasa las diferentes políticas educativas en relación a la incorporación de las TIC en la educación, cuya idea central es la necesaria convergencia de factores políticos, económicos, tecnológicos, pedagógicos, sociales y administrativos. Así mismo, el estudio realizado por Meneses, Fábregues, Jacovkis y Rodríguez-Gómez (2014) examina las políticas autonómicas de integración de TIC en los centros educativos de nuestro país.

Uno de los proyectos más relevantes es el programa "Escuela 2.0" que ha dotado a numerosas aulas de Primaria y Secundaria de ordenadores y pizarras digitales (GarcíaValcárcel, Basilotta y López, 2014). En materia de PDIs destacan investigaciones internacionales como las de Bell (2002), Glover y Miller (2001), Lewin, Scrimsaw, Somekh y Haldane (2009) para los centros ingleses, Maher, Phelps, Urane y Lee (2012) para centros del Reino Unido, Dinamarca, México y Australia, etc. En el contexto español mencionamos el "Iberian Research Project" (Gallego, Cacheiro y Dulac, 2009) y el grupo DIM-UAB dirigido por Marqués, que investigan la integración de la PDI en las aulas. Vemos, por tanto, un interés creciente a nivel nacional e internacional de acercar las PDIs a los centros educativos.

En general, las TIC han llegado, no sólo para quedarse, sino para formar parte de un proceso de enseñanza-aprendizaje innovador y adaptado a las nuevas necesidades. Para ello, es necesario preparar a un alumnado capaz de gestionar la inmensa cantidad de información que recibe a diario (OEl, 2013), por lo que se hace necesario un trabajo en red de todos los agentes implicados y una adecuada integración de las tecnologías en el currículum. El equipo directivo debe liderar y apoyar iniciativas sobre la introducción de tecnología en el centro, que junto con el resto de docentes, serán elementos claves para una integración exitosa (González, Recamán y González, 2013). En este sentido Monge (2013, p. 123) resalta que dicha integración "ha de realizarse en el marco de un proyecto pedagógico que le dé sentido y significación y que le legitime para decidir sobre cuándo, cómo y porqué del uso de un determinado medio o tecnología". En esta línea la Teoría de la Educación y la Pedagogía activa tienen mucho que reflexionar y aportar en un contexto de enseñanza-aprendizaje compartido, interactivo y competencial.

Además, se debe garantizar que se planifiquen adecuadamente en estos proyectos los estándares que plantea UNESCO (2008) sobre competencias TIC para docentes que requieren de actuaciones planificadas y compromisos compartidos:

- Garantizar el conocimiento y desarrollo de nociones básicas en TIC, tales como el manejo de recursos como ordenadores, PDIs, "tablets", uso de recursos digitales como "power point", mapas conceptuales, "blog", etc. El profesor de hoy debe conocer las herramientas de la web 2.0 y saber utilizarlas de manera didáctica en sus clases (Cebrián de la Serna, 2009).

- Profundización del conocimiento que permita a profesores y a alumnos incrementar y perfeccionar el manejo de la tecnología para su desarrollo académico y personal. Para ello se requiere saber buscar información, filtrarla, analizarla, etc. (Monereo y Badia, 2012).

- Desarrollo de la generación de conocimiento que implica la creación de materiales, diseño de recursos, desarrollo del pensamiento crítico, reflexivo, etc. 
Estos estándares deben contextualizarse dentro de proyectos educativos basados en paradigmas constructivos y, tal como indica Aparici y Silva (2012), apoyados también en la pedagogía de la interactividad, en la que todos los participantes del proceso de enseñanzaaprendizaje se alimenten de la comunicación, intercambio de opiniones y trabajo colaborativo.

\section{Metodología}

Con la finalidad de incorporar las tecnologías a la educación de los centros públicos de Educación Infantil y Primaria de Alcorcón y contribuir, de esta manera, a la mejora de la calidad, en el curso 2010/2011 su Ayuntamiento les dotó con 400 PDIs (318 Smart y 82 InterWrite). La inversión económica fue grande por lo que es conveniente analizar si esa apuesta económica ha permitido obtener los resultados esperados. Para ello, se ha realizado un estudio estadístico de análisis descriptivo y correlacional utilizando el programa SPSS en su versión 21 y una trascripción tradicional de las entrevistas realizadas.

\section{Participantes}

El muestreo utilizado fue probabilístico simple y la muestra con la que contamos es: 133 profesores, 776 alumnos de Educación Primaria, 252 familias y 21 coordinadores TIC (uno por cada centro educativo). El $26.3 \%$ de los maestros encuestados eran hombres y el $73.7 \%$ mujeres. La distribución por cursos es similar entre el último curso de infantil ( 5 años) y los seis cursos de primaria.

\section{Objetivos de la investigación}

El objetivo general de la investigación es conocer el impacto inicial y actual de las pizarras digitales interactivas en los centros participantes desde la percepción de profesores y coordinadores TIC. Ambos se convierten en los principales agentes de cambio del proceso de integración e innovación de TIC en las aulas.

También interesa conocer como objetivo específico, cuáles son las principales ventajas e inconvenientes implícitos en la integración de las PDIs dentro del proceso de enseñanzaaprendizaje.

\section{Diseño de la investigación}

El presente trabajo consta de dos fases, una inicial centrada en la visión del profesorado encuestado y otra actual centrada en la visión de los coordinadores TIC entrevistados. La metodología utilizada es de carácter mixto (cuantitativa- cualitativa) y nos permitirá triangular los resultados de los agentes implicados en el estudio: coordinadores TICprofesores, padres y alumnos.

\section{Instrumentos de recogida de información}

Los instrumentos utilizados en la investigación completa han sido:

- Grupo de discusión realizado con los coordinadores TIC al comienzo de la implantación, en el que trataron temas como las expectativas iniciales, necesidades planteadas, problemática encontrada, actitudes iniciales, etc. 
- Registro de observación utilizado en las visitas a las aulas, donde se observaron aspectos como los tipos de actividades realizadas en las PDIs, nivel de manejo por parte de profesores y alumnos, actitud de profesores y alumnos, uso de otras herramientas digitales en la PDI...

- Cuestionarios semi-estructurados, previamente validados por expertos y dirigidos a alumnos, profesores y familias.

- Entrevistas a profesores, alumnos y coordinadores TIC sobre el nivel de acogida del nuevo recurso y su influencia en el proceso de enseñanza-aprendizaje.

En relación a la primera fase, el cuestionario dirigido al profesorado fue distribuido a finales del curso 2010/2011, y los aspectos más significativos son:

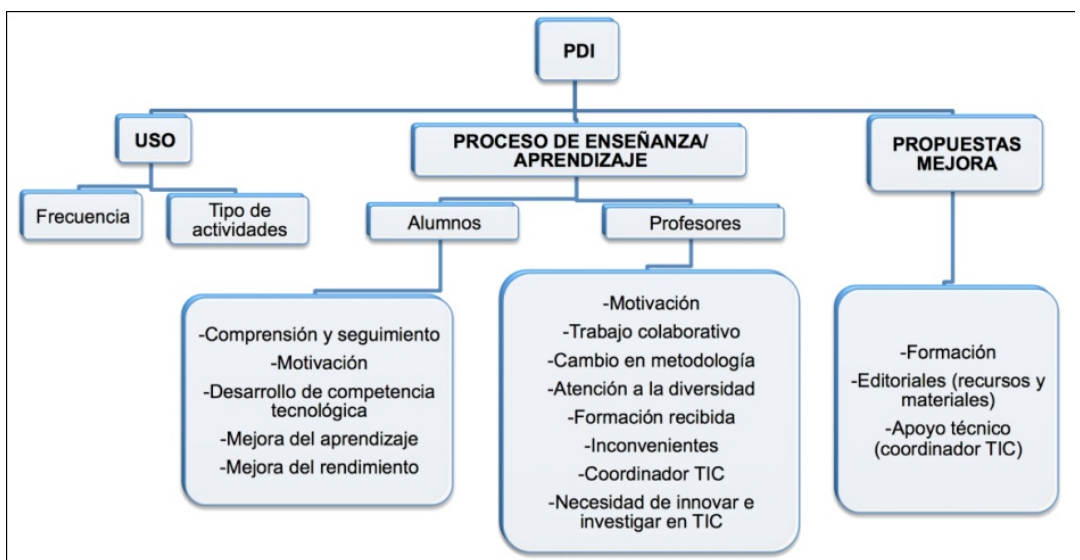

Figura 1: Bloques y variables analizadas en el cuestionario a profesores. Elaboración propia.

En la segunda fase, se realizaron entrevistas individuales a los coordinadores TIC de los centros a finales del curso 2013/2014 en relación a la formación continua sobre PDI, mejoras en la motivación de profesores y alumnos, incremento de funciones, valoración general del uso de la PDI y del recurso como favorecedor del aprendizaje del alumno.

\section{Procedimiento}

A continuación se presentan las tres fases de investigación distribuidas en varios cursos académicos:

- Fase inicial de diagnóstico: el objetivo fundamental es realizar la primera toma de contacto con el proceso de implantación de PDIs en los centros, conocer la situación de partida, actitudes y expectativas de los centros, delimitar objetivos y planificar actuaciones.

- Fase de diseño y desarrollo de la investigación: Visitas a los centros participantes y distribución de los cuestionarios a los destinatarios, realización de entrevistas a profesores y alumnos y uso de los registros de observación en las visitas a las aulas. 
- Fase de evaluación final: en esta fase se analizarán los datos obtenidos en los diferentes instrumentos de recogida de información, se llevarán a cabo las entrevistas finales a los coordinadores TIC para conocer la evolución de la PDI en estos centros, redacción del trabajo de investigación, etc.

Tabla 1.

Cronograma de la investigación

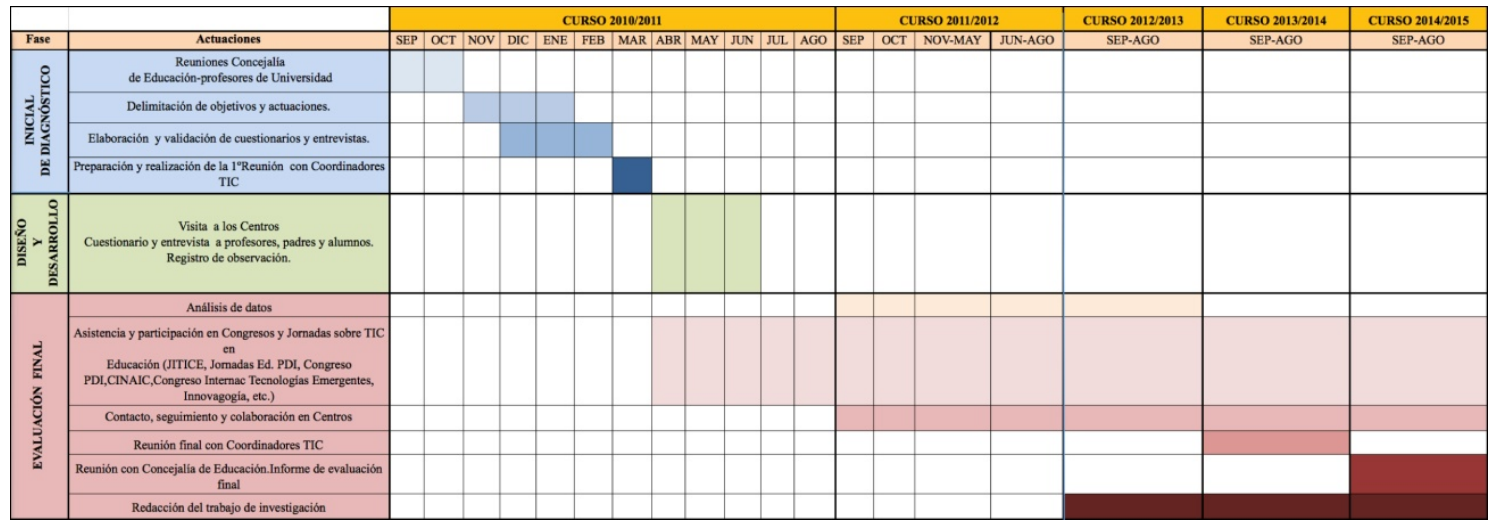

\section{Resultados}

Seguidamente se presentan algunos resultados obtenidos en las fases de implantación y evolución de las PDIs en relación al uso de las TIC y PDI, influencia en el proceso de enseñanza-aprendizaje y propuestas de mejora para su implementación.

\section{Fase inicial}

Se realizó un análisis descriptivo de las variables más relevantes en relación al profesorado y resaltamos que la mayor parte de ellos (75.2\%) afirmaron utilizar "mucho" o "a menudo" la PDI. Únicamente un $2.3 \%$ de los profesores afirmaron no haberla usado nunca. Aún así se puede afirmar que la acogida inicial fue positiva.

En cuanto al tipo de actividades que suelen realizar en la PDI, un 68.4\% presenta en "muchas ocasiones" o "a menudo" materiales audiovisuales, el 36.1\% afirman que los alumnos son los que realizan actividades en la PDI, destacando un $28.6 \%$ que reconocen que los alumnos no la suelen utilizar y que sólo la utilizan ellos. Éste sería uno de los errores frecuentes, pues se trata de que ambos saquen provecho a la herramienta. Al cruzar los datos sobre las dos herramientas digitales más utilizadas en la PDI vemos que casi un 30\% consultan webs, un 13\% presentaciones en "power point", "you tube", "blogs", "wikis", etc. Además, el $57.1 \%$ del profesorado opina que la PDI favorece "mucho" el seguimiento y comprensión de las asignaturas.

Por otra parte, numerosas investigaciones (Campregher, 2010; Cascales y Laguna, 2014; Domingo y Marqués, 2013; Fernández, 2013; Lan y Hsiao, 2010; Toledo y Sánchez, 2013) resaltan que la PDI favorece la motivación de profesores y alumnos. En este sentido, casi un $73 \%$ del profesorado piensa que les motiva "mucho" y "a menudo", y ningún profesor 
afirma que la PDI no motiva nada. Con respecto al alumno, frente a un $86.4 \%$ que están "muy" motivados tan solo uno de ellos opina que no favorece su motivación (0.8\%), por lo que los datos, en su mayoría, siguen siendo positivos. Estos resultados se corroboran en la European Commission (2013) puesto que

"una gran mayoría de directores de escuela y profesores están de acuerdo sobre la importancia del uso de las TIC en las diferentes actividades de aprendizaje, así como sobre el impacto positivo del uso de las TIC en la motivación y el rendimiento de los estudiantes, y en un orden transversal y superior, en las habilidades de pensamiento"(p.12).

Otro elemento que se trabaja con las TICs es el desarrollo de la competencia tecnológica y digital muy útil ante el aluvión de información que solemos recibir. Se produce, en ocasiones lo que Aguaded (2014) denomina infoxicación de los medios. Información que deberá ser capaz de organizar y comunicar de manera apropiada, por lo que el desarrollo simultáneo con otro tipo de competencias básicas es muy importante (Bustos, 2011; ITE, 2011; OCDE, 2005). En este sentido un $87.2 \%$ del profesorado opina que sus alumnos desarrollan "mucho" y "a menudo" estas competencias.

Por otra parte interesa comprobar si la PDI realmente mejora el aprendizaje de nuestros alumnos, resaltando un 74\% del profesorado que indica que mejora "mucho" y "a menudo", y dos profesores (1.5\%) que consideran que no mejora el aprendizaje. Un 5.3\% no saben si realmente mejora el aprendizaje, pues quizá los resultados son más difícilmente comprobables que factores como la motivación y el comportamiento que son fácilmente visibles. Al cruzar las variables "profesores que usan con gusto la PDI" con "favorece la mejora del aprendizaje" vemos que aquellos que la utilizan mucho tienen mejor opinión al respecto que los que no la usan o la usan alguna vez.

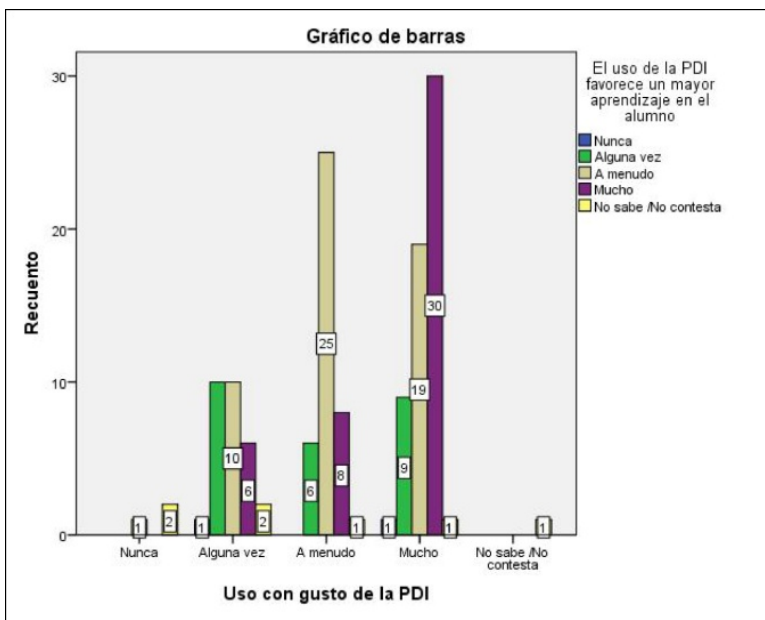

Gráfico 1. Mejora del aprendizaje del alumno tras el uso de la PDI

En relación a los resultados académicos, un $41.4 \%$ de los profesores opina que en "algunas ocasiones" el uso de la PDI mejora los resultados académicos de sus alumnos, un 30.8\% "a menudo", un $13.5 \%$ cree que lo mejora "mucho" y un $7.5 \%$ opina que "nunca" lo mejora, por lo que las perspectivas de éxito no se han cumplido totalmente aunque son positivas. Al 
cruzar las variables "profesores que usan con gusto la PDI" con "favorece la mejora de los resultados académicos" volvemos a percibir una mejora en la percepción de aquellos que más las utilizan.

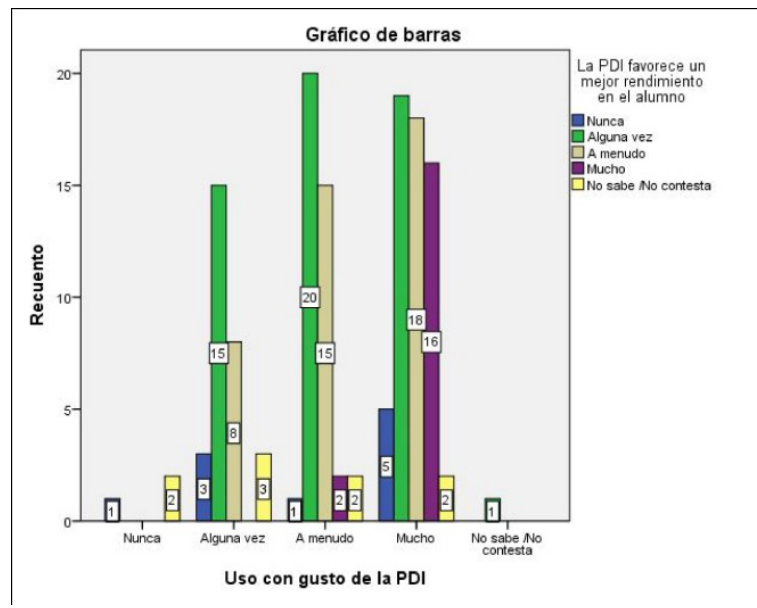

Gráfico 2. Mejora del rendimiento del alumno tras el uso de la PDI

Tal y como manifiestan algunos profesores en el apartado "observaciones", estos resultados pueden ser debidos a la necesidad de haber usado durante más tiempo la PDI para poder valorar estos dos aspectos.

Así mismo, tradicionalmente les evaluamos a través de exámenes, sin embargo, las competencias y destrezas que el alumno desarrolla mientras utiliza tecnología es difícilmente medible con este tipo de pruebas. Por lo tanto, en general el problema se encuentra en cómo el profesor entiende y lleva a cabo la evaluación de sus alumnos, por lo que debemos replantearnos qué entendemos por "competencia” y cómo queremos evaluarlas adecuadamente (Prats, 2013). Todo ello acompañado por supuesto de una metodología que les permita trabajar competencias y alfabetizarles desde una perspectiva mediática, digital, multimodal, crítica y funcional (Gutiérrez y Tyner, 2012).

En relación al trabajo colaborativo favorecido por el uso de las PDIs, un $44.1 \%$ piensan que lo favorece "alguna vez", seguido de un $33.1 \%$ que indica que lo favorece "a menudo". Debido a que estos profesores se encontraban en su primer año de uso, es lógico que un $5.3 \%$ nunca haya intercambiado experiencias con otros compañeros. El hecho de trabajar con TICs, intercambiar experiencias, crear tus propios recursos educativos abiertos y quererlos compartir en comunidades o foros favorece el espíritu colaborador del profesorado y prácticas más enriquecedoras (Gatica y Valdivia, 2013; Tosato, Carramolino y Rubia, 2014). De esta manera, algunos aspectos tales como "partir del compromiso con la tarea y sus objetivos, tanto en el proceso como en el producto, la responsabilidad, el liderazgo compartido y el desarrollo de habilidades interpersonales", se vuelven imprescindibles en el proceso de enseñanza-aprendizaje (Torras-Virgili, 2013: 168-169). 
Pero, como la tecnología por sí sola no mejora el aprendizaje ni la enseñanza, el desarrollo de una buena metodología adaptada a las TIC es clave. En este sentido, casi un 70\% del profesorado destaca haber introducido ("mucho" o "a menudo") algún cambio metodológico tras la introducción de la PDI en el aula, seguidos de casi un $25 \%$ que reconocen haber cambiado su metodología en "alguna ocasión" y de un $1.5 \%$ que niegan haber cambiado su metodología. Este último dato (junto con el 3.8\% que no contestan) preocupa especialmente pues el profesor debe asociar cierto cambio metodológico al uso de tecnología. Esto, obviamente, no sería innovar sino seguir reproduciendo metodología tradicional con recursos diferentes. A tenor de lo expuesto, un profesor con una buena metodología llevará a cabo una eficaz enseñanza con pizarras digitales si sigue los siguientes principios: ser competente, tener una buena organización, trabajar desde y para la interactividad, ser flexible, constructivo, ser abierto de mente, desarrollar la capacidad de compartir y trabajar con una buena planificación (Betcher y Lee, 2009).

Por otro lado, la PDI favorece "mucho" o "a menudo" la atención a la diversidad (74.4\%), lo que demuestra que es una ayuda para todos los estudiantes (Fernández, 2013).

En cuanto a la formación inicial recibida, el mayor porcentaje de profesores (35.3\%) la valoraron como "nada" útil ni suficiente, seguido de un $34.6 \%$ que consideran que "alguna vez" les ha resultado útil. Sólo el $7.5 \%$ consideraron fue muy útil. Estos datos nos dan pistas incuestionables de que hay que continuar invirtiendo en formación si queremos conseguir buenos resultados.

Así mismo, el coordinador TIC de los centros es valorado muy positivamente por casi un 70\% de los encuestados resaltando su asesoramiento y seguimiento continuo. La visión, apoyo y feedback que proporciona estos profesores ayuda enormemente al proceso de integración de las tecnologías en los centros.

La necesidad de seguir investigando e innovando sobre TICs aplicadas a la educación es representada por cerca del $86.5 \%$ del profesorado, aunque 7 profesores consideran que no es necesario o simplemente no contestan, lo cual nos parece preocupante, pues hoy en día es fundamental adaptar las aulas a los avances que se producen socialmente. Además, a pesar del cada vez mayor acceso a recursos tecnológicos "parecen todavía relativamente escasos los usos educativos (de las TIC) realmente innovadores" (Fernández-Díaz y Calvo, 2013: 121). También, es importante entender la escuela como "un espacio programado pedagógicamente para educar, cuyas posibilidades de innovación son continuas cada día en cada acción concreta" (Colom, Castillejo, Pérez, Sarramona y Touriñán, 2012: 27). Por ello, los docentes deben aprovechar las tecnologías para desarrollar la creatividad e innovación en su quehacer diario.

Por otra parte, destacamos inconvenientes como "algunas" resistencias iniciales al recurso (42.9\%) y una "muy alta" inversión de tiempo por parte del profesor (68.4\%), lo que en ocasiones es lógico en su momento inicial (García-Valcárcel, Basilotta y López, 2014). "Los maestros necesitan tiempo para tener confianza y ser competentes en el uso de las herramientas antes de utilizarlas de formas nuevas en sus aulas" (Lee, 2010: 137).

Como propuestas de mejora en el uso de la PDI destacamos que la mayoría de las observaciones realizadas por el profesorado encuestado trataban sobre la necesidad de 
recibir más formación en TIC en general y sobre la PDI en particular. La formación del maestro en activo, se convierte en un elemento trascendental en la integración de las PDIs en las aulas, pero también la formación del futuro maestro cobra especial importancia en este proceso (Cabero, 2014; Naval y Arbués, 2012). De hecho, desde los planes de estudio del Grado de Educación Infantil y Grado de Educación Primaria se debe incorporar la adquisición de competencias TIC y estrategias didácticas para aprender a gestionar la información. "La competencia informacional se manifiesta, por tanto, como un requerimiento para la formación que se debe ofrecer en los Centros de Educación de Infantil y Primaria (CEIP) desde la formación inicial en la Universidad" (Negre, Marín y Pérez, 2013: 2).

Otras dificultades manifestadas por los profesores fueron:

- “A veces los problemas técnicos interrumpen la actividad en la PDI” (profesor 11)

- "Es una herramienta muy útil pero que necesita internet para sacarle el máximo rendimiento" (profesor 18)

- “Necesitamos ordenadores más potentes" (profesor 19)

- "Cuando las editoriales preparen todas las actividades para pizarras, las usaremos más, mientras tanto no porque no hay tiempo para prepararlas" (profesor 83)

- "Nos han regalado unos excelentes automóviles de lujo pero no tienen combustible y muchos profesionales no tenemos el carnet para conducirlos" (profesor 85).

Por otra parte varios manifiestan la necesidad de que existan técnicos informáticos en los centros para solucionar problemas de software y hardware, y de esta manera el coordinador TIC se dedique a impartir formación y diseñar proyectos de innovación TIC.

Fase actual

Esta fase se caracteriza por el estudio del impacto actual de las PDIs a través de las percepciones de los coordinadores TIC entrevistados tres años después de su implantación.

En primer lugar es interesante destacar que la mayoría de ellos asumen estas funciones desde hace unos 5-10 años, salvo excepciones, por lo que han sido protagonistas desde su implantación.

En relación a la formación continua sobre la PDI la mayoría sí han recibido algún tipo de formación a lo largo de estos últimos años, así como de otras herramientas TIC (sobre todo blogs y búsqueda de recursos digitales). Esto lo corroboran cuando indican que "no ha habido una formación (inicial) que haya merecido la pena pero nos hemos apañado nosotros mismos y luego lo que cada uno haya trasteado" (profesor 1). En relación a la mejora de la motivación del profesorado se percibe una mejora significativa: "Ha habido un cambio bestial, aquí había gente que no sabía ni encender un ordenador, y ahora todos utilizan la PDI, en menor o mayor medida" (profesor 1). "Un salto cualitativo fue que las editoriales hicieses libros digitales en formato pdf. Esto ha generado que los profesores estén tranquilos y sientan seguridad por el apoyo que supone" (profesor 5 ).

La motivación del alumno hacia la PDI también es positiva destacando la opinión del profesor 9 cuando afirma que "para un alumno, una superficie táctil no es nada que no sea algo cotidiano, con lo cual lo que es motivador es el diseño de la actividad docente". Todos 
los coordinadores han percibido un incremento en sus funciones desde la implantación de las PDIs, sobre todo a nivel técnico. "Es una herramienta que en el momento en que falla hay que ponerle solución cuanto antes, no se puede esperar" (Profesor 3). Esto lo ratifican Espuny, Gisbert, Coiduras y González (2012: 6) cuando afirman que "su función ha cambiado en los últimos años ante las exigencias de una escuela 2.0., pasando de una actividad más centrada en el hardware, a ocuparse actualmente de la dinamización de las TIC".

En relación a si el uso de la PDI favorece una mejora en los resultados académicos del alumno, todos ellos se muestran dubitativos ante la pregunta. "El rendimiento como tal no sé si lo podría afirmar, lo que sí se ha mejorado es el acceder a la información, el motivar al alumno para buscar información, todo eso sí" (profesor 2). "Pues no creo que dependa del recurso, sí depende del profesional que utilice el recurso, de cómo se utilice, pero no es determinante. Mejora el desarrollo de destrezas y de competencias, sobretodo de competencia digital" (profesor 5). "Es más motivadora, los implica más y otra forma de presentación de los contenidos, pero establecer una relación directa con la mejora académica es complicado" (profesor 6). Los coordinadores se muestran más cautelosos en esta fase final que los profesores encuestados en la fase inicial.

En relación a los inconvenientes destacan problemas técnicos en relación a la conexión wifi, compra de ordenadores nuevos por fallos en los "notebook", compra de dispositivos para audio, etc. Sin embargo y, en general, la valoración que realizan sobre el uso que se hace actualmente en sus centros de la PDI es notable alto. Coincidiendo también con la valoración general que hacen de la pizarra digital como recurso favorecedor del aprendizaje general del alumno. En definitiva se pueden identificar varios factores influyentes en el uso adecuado de la PDI, sin los cuales la tecnología en sí no tendría el sentido pedagógico que requiere.

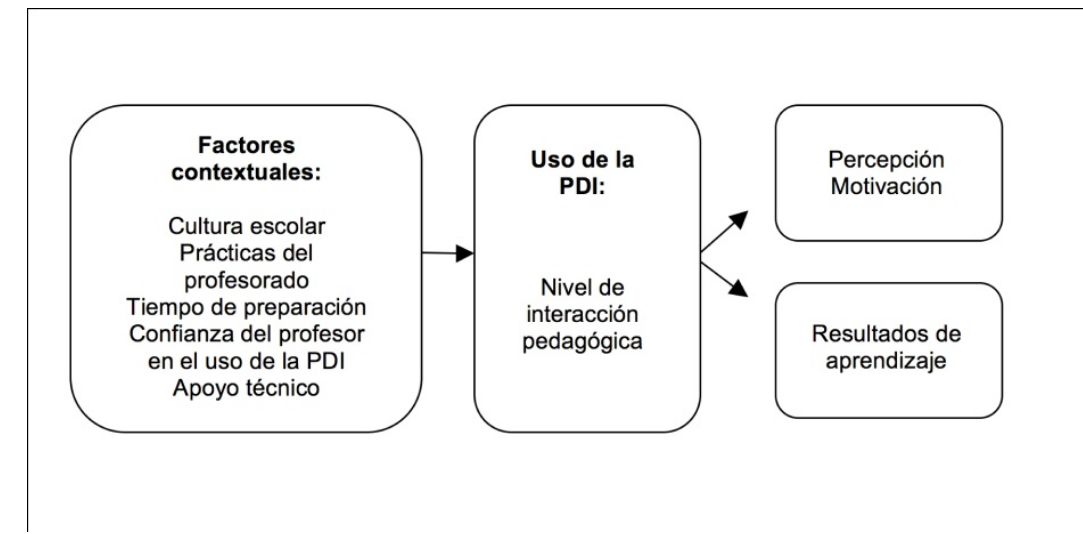

Figura 2: Factores contextuales influyentes en el uso adecuado de la PDI en el aula. Di Gregorio y SobelLojeski (2010: 257)

Entendemos, por tanto, que no sólo los factores contextuales influyen en menor o mayor medida en el uso adecuado de los recursos digitales, sino también los factores personales que son determinantes para un mejor aprovechamiento de aquellos elementos y herramientas que nos rodean. 


\section{Discusión y conclusiones}

Tras analizar las percepciones e impacto de la pizarra digital en el proceso de enseñanzaaprendizaje, tanto en su momento inicial como en la actualidad, podemos concluir que hoy en día se requiere cada vez más profesores expertos en sus materias pero también competentes en tecnología aplicada a la educación. Sabemos que el hecho de que el centro cuente con tecnología en sus aulas no implica que se utilice, por lo que para conseguir buenos resultados, una vez más, el factor clave es la formación del profesorado (Di Gregorio y Sobel-Lojeski, 2010; Domingo y Marqués, 2013; Gandol, Carrillo y Prats, 2012; Marqués, 2009; entre otros). Para ello, es imprescindible que el docente esté motivado, reciba formación técnica y pedagógica continuada y que cuente con infraestructura y recursos adecuados. La PDI se convierte entonces en una ventana abierta al mundo que nos rodea y nos permite interactuar, comunicarnos y aprender.

Además, es un recurso ideal para combinar con la enseñanza tradicional pero al mismo tiempo descubrir las grandes posibilidades innovadoras que posee y avanzar hacia metodologías más constructivas e interactivas centradas en el alumno y no tanto en el profesor o en el recurso en sí (Gustavo, 2014; Fernández, 2013). En este sentido, los docentes deben descubrir las posibilidades que ofrece una pedagogía de la interactividad frente a la reproducción de metodologías tradicionales. $Y$ recordando que lo que se pretende realmente cuando trabajamos con TICS es favorecer cierta interactividad con los recursos e interacción con las personas.

Siguiendo esta idea, la colaboración que se puede desarrollar en el aula gracias a estos recursos se convierte, según Churches (2009), en una habilidad imprescindible facilitadora de otras habilidades cognitivas como comprender, recordar, aplicar, analizar, crear y evaluar. Sin embargo esto requiere que el maestro se replantee su metodología y evaluación, pues si su manera de enseñar y evaluar contemplara más aprendizajes competenciales probablemente los resultados de los alumnos también serían más significativos (Domingo, 2011; Domingo y Marqués, 2011, 2013).

Según hemos podido comprobar en la investigación presentada, en general los profesores están realizando un esfuerzo por incorporar esta nueva herramienta. Bien es verdad que la inversión (sobre todo inicial) de tiempo del maestro es uno de los factores más influyentes en su implementación. Sin embargo, se resaltan ventajas altamente valoradas por el profesorado como: mayor interacción entre alumnos y profesores, aumento de la motivación e interés en las clases, desarrollo de competencias, etc. Además, tal y como indican Real, Cascales y Marcos (2012: 1) "la pizarra digital es un recurso de grandes posibilidades educativas y puede ayudar decisivamente a la renovación pedagógica, en tanto que se adapta a los estilos de aprendizaje de todos los alumnos". La relación entre el uso de la PDI y su adaptación a los diferentes estilos cognitivos de aprendizaje ha sido investigada por autores como Campregher (2010).

Por otro lado, la relación directa entre la PDI y el aprendizaje general del alumno (competencias, capacidades...) es buena, sin embargo su correspondencia con la mejora de los resultados académicos no es tan clara. Aún así, es un recurso bien valorado dentro del proceso de enseñanza-aprendizaje. 
Como líneas de trabajo futuras se plantea correlacionar las percepciones de los profesores encuestados con la visión de alumnos y familias para lograr una investigación más completa. Así mismo, es interesante difundir e intercambiar experiencias de aquellos centros avanzados en el uso de las tecnologías, todo ello para compartir prácticas y mejorar las relaciones entre ellos. Centros como por ejemplo, el CEIP Santo Domingo ha sido nombrado en 2014 el $1^{\circ}$ colegio público de innovación tecnológica de Alcorcón, o el CEIP Joaquín Costa también nombrado en el curso 2013/2014 centro tecnológico y elegido para formar parte del Proyecto de Innovación de metodología educativa a través de "tablets" (Proyecto Samsung Smart School).

Por otro lado, se elaborará un informe detallado con los principales resultados obtenidos del impacto inicial y actual de la PDI que se proporcionará a los centros participantes y a la Concejalía de Educación para conocer los principales resultados y las necesidades actuales.

La investigación no está exenta de limitaciones. La principal fue que, el proceso de implantación del recurso, la formación y la familiarización fue muy dispar entre los centros, por lo que hubiera sido interesante partir de situaciones similares para comparar resultados en iguales condiciones.

No obstante, la principal aportación de este trabajo es presentar un caso concreto de implantación de PDIs en centros de características diversas, conocer los principales inconvenientes para intentar hacerlos frente y analizar factores favorecedores para una adecuada integración en las aulas.

En conclusión, el estudio de la literatura refleja la evidencia de que la simple presencia de recursos tecnológicos en las aulas, como es el caso de la PDI, no es suficiente por sí sola para mejorar la enseñanza o el aprendizaje. Didáctica, pedagogía y tecnología se complementan para dar respuesta a las necesidades de nuestra educación actual y en este entorno el profesor se convierte en la piedra angular del proceso. Esta idea la defiende Duan (2010: 147) cuando resalta que "el tener en cuenta el potencial de la PDI al final depende de nuestros maestros: cómo entender la naturaleza de la enseñanza interactiva; elegir el tipo de estrategias pedagógicas y métodos de enseñanza, cómo diseñar actividades de enseñanza esclarecedoras..."

Al lado del maestro sin duda, el coordinador TIC es su principal apoyo. En definitiva, debemos conseguir aulas con tecnologías pero apoyadas de mucha pedagogía, y que nos ayuden a enfrentarnos al desafío que nos presentan las TIC. Para ello, debemos caminar hacia un nuevo paradigma, el tecnológico, en el que sea necesaria una adecuada formación técnica y pedagógica pero también voluntad de cambio en nuestras metodologías y evaluación. Aspectos, que en ocasiones ocupan un segundo plano y que, sin embargo, son el motor que mueve cualquier innovación. 


\section{Bibliografía}

Aguaded, J.I. (2014). Desde la infoxicación al derecho de la comunicación. Comunicar, 21 (42), 7-8.

Aparici, R., \& Silva, M. (2012). Pedagogía de la interactividad. Comunicar, 19 (38), 51-58.

Area, M. (2006).Veinte años de políticas institucionales para incorporar las tecnologías de la información y comunicación al sistema escolar. En J.M. Sancho (Coord). Tecnologías para transformar la educación (pp. 199-232). Madrid: AKAL/U.I.A.

Bell, M.A. (2002). Why Use an Interactive Whiteboard? A Baker's Dozen Reason! Teacher.net.

Betcher, C., \& Lee, M. (2009). The interactive whiteboard revolution. Melbourne, Australia:ACER Press.

Bristish educational communications and technology agency (BECTA) (2004). Getting the most from your interactive whiteboards. A guide for primary schools.

Bustos, E. Mº. (2011). Las TIC en el aula: de la pizarra digital al aprendizaje por competencias. Revista digital de innovación y experiencias educativas, (38).

Cabero, J. (2014). Formación del profesorado universitario en TIC. Aplicación del método Delphi para la selección de los contenidos formativos. Educación XX1, 17 (1), 111-132.

Campregher, S. (2010). The Future of Education, Effects of the interactive whiteboard (IWB) in the Classroom Experimental Research in Primary School. International Conference University of Bolzano (Italy).

Cascales A. \& Laguna, E. (2014). Una experiencia de aprendizaje con la pizarra digital interactiva en Educación infantil. Píxel-Bit. Revista de Medios y Educación. 45, 125-136.

Cebrián de la Serna, M. (Coord.), SÁNCHEZ, J. y PALOMO, R. (2009). El impacto de las TIC en los centros educativos. Ejemplo de buenas prácticas. Barcelona: Síntesis.

Churches, A. (2009) Taxonomía de Bloom para la era digital. Educational Origami.

Colom. A.J., Castillejo, J.L., Pérez, P. Mº., \& Touriñán, J. M. (2012). Creatividad, educación e innovación: emprender la tarea de ser autor y no sólo actor de sus propios proyectos. Revista de investigación en educación, 1 (10), 7-29.

Di Gregorio, P. \& Sobel-Lojeski, K. (2010). The effects of interactive whiteboards (IWBS) on student performance and learning: a literature review. J.Educational Technology Systems, 38 (3), 255-312.

Domingo-Coscollola, M. (2011). Pizarra Digital Interactiva en el aula: Uso y valoraciones sobre el aprendizaje. Estudios sobre educación (ESE), 20, 99-116.

Domingo, M., \& Marqués, P. (2011). Aulas 2.0 y uso de las TIC en la práctica docente. Comunicar, 19 (37), 169-175.

Domingo, M. \& Marqués, P. (2013). Experimentación del uso didáctico de la pizarra digital interactiva (PDI) en el aula: Plan formativo y resultados. Enseñanza y Teaching: Revista interuniversitaria de didáctica, 31 (1), 91-108. 
Duan, Y. (2010). Teaching interactively with Interactive Whiteboard: Teachers are the key. Networking and Digital Society (ICNDS), 2nd International Conference on,1, 144 -147.

Espuny, C., Gisbert, M., Coiduras, J.L \& González, J. (2012). El coordinador TIC en los centros educativos: funciones para la dinamización e incorporación didáctica de las TIC en las actividades de aprendizaje. Pixel-Bit: Revista de medios y educación, (41), 7-18.

European Commission (2013). Survey of schools: ICT in education final study report benchmarking access, use and attitudes to technology in Europe's schools.

Fernández, R.R. (2013). La pizarra digital interactiva como una de las tecnologías emergentes en la enseñanza actual. 3 Ciencias TIC. 5, 1-15.

Fernández-Díaz, E. \& Calvo, A. (2013). Estrategias para la mejora de la práctica docente. Una investigación-acción colaborativa para el uso innovador de las TIC. Revista Electrónica Interuniversitaria de Formación del Profesorado (REIFOP), 16 (2), 121-133.

Gallego, G., Cacheiro, M.L. \& Dulac, J. (2009). La pizarra digital interactiva como recurso docente. Revista Electrónica Teoría de la Educación: Educación y Cultura en la Sociedad de la Información, 10 (2), 127-145.

Gandol, F., Carrillo, E. \& Prats, M.A. (2012). Potencialidades y limitaciones de la pizarra digital interactiva. Una revisión crítica de la literatura. En Pixel-Bit. Revista de Medios y Educación, (40), 171-183.

García-Valcárcel, A., Basilotta, V. \& López, C. (2014). Las TIC en el aprendizaje colaborativo en el aula de Primaria y Secundaria. Comunicar, 21, (42), 65-74.

Gatica, N. \& Valdivia, J. (2013). La Pizarra Digital Interactiva y la Web 2.0: ambientes digitales que se sustentan con la teoría del constructivismo social. En D. Gallego, y M. Álvarez (Ed.), Capacitación y Gestión del conocimiento a través de la Web 2.0 (pp. 232-255). Madrid: Dykinson.

Glover, D., \& Miller, D. (2001). Running with Technology: The Pedagogic Impact of the Large Scale Introduction of Interactive Whiteboards in One Secondary School. Journal of Information Technology for Teacher Education, 10, 257-276.

González, L.M, Recamán, A., \& González, C. (2013). La dimensión colaborativa con TIC en la dirección de centros. Revista Electrónica Interuniversitaria de Formación del Profesorado (REIFOP), 16 (1), 147-162.

Gustavo, M. (2014). La enseñanza en un mundo en transformación: el uso de las Tecnologías de la Información y la Comunicación. Virtualidad, Educación y Ciencia, 9 (5), 90-97.

Gutiérrez, A. \& Tyner, K. (2012). Educación para los medios, alfabetización mediática y competencia digital. Comunicar, 19 (38), 31-39. (DOI: http://doi.org/tkd ).

Instituto de Tecnologías Educativas. Departamento de Proyectos Europeos. (2011). Competencia digital. ITE: Ministerio de Educación.

Lan, T.S. \& Hsiao, T. Y. (2010). A Study of Elementary School Students' Viewpoints on Interactive Whiteboard. American Journal of Applied Sciences, 8 (2), 172-176. 
Lee, M. (2010) Interactive whiteboards and schooling: the context. Technology, Pedagogy and Education, 19 (2), 133- 41.

Lewin, C., Scrimshaw, P., Somekh, B., \& Haldane, M. (2009). The impact of formal and informal professional development opportunities on primary teachers' adoption of interactive whiteboards. Technology, Pedagogy and Education, 18 (2), 173-185.

Maher, D., Phelps., R. , Urane, N. \& Lee, M. (2012). Primary school teachers' use of digital resources with interactive whiteboards: The Australian context. Australasian Journal of Educational Technology, 28 (1), 138-158.

Marqués, P. (2009). Las claves del éxito para el uso de la pizarra digital, la web 2.0 y las aulas 2.0. Grupo DIM.

Meneses, J., Fàbregues, S., Jacovkis, J., \& Rodríguez-Gómez, D. (2014). La introducción de las TIC en el sistema educativo español (2000-2010): un análisis comparado de las políticas autonómicas desde una perspectiva multinivel. Estudios sobre educación, 27, 63-90.

Monge, C. (2013). La pizarra digital: las aulas, espacios con tizas y punteros. Anales: Anuario del centro de la UNED de Calatayud, 1 (19), 119-135.

Monereo, C. \& Badia, A. (2012). La competencia informacional desde una perspectiva psicoeducativa: enseñanza basada en la resolución de problemas prototípicos y emergentes. Revista Española de Documentación Científica, 75-99.

Naval, C. \& Arbués, E. (2012). La alfabetización mediática de los futuros profesores de educación primaria y secundaria. XXXI Seminario Interuniversitario de Teoría de la Educación (XXXI SITE). Sociedad del Conocimiento y Educación. Coordinado por García Aretio, UNED, Plasencia 11 y 14 de noviembre de 2012.

Negre, F., Marín, V.I., \& Pérez, A. (2013). Estrategias para la adquisición de competencias informacionales en la formación inicial de profesorado de primaria. Revista Electrónica Interuniversitaria de Formación del Profesorado (REIFOP), 16 (2).

OCDE (2005). The definition and selection of key Competences. Executive Summary. Madrid: Ministerio de Educación, Cultura y Deporte.

OCDE (2013). Los desafíos de las tecnologías de la información y las comunicaciones en la educación. Madrid: Ministerio de Educación, Cultura y Deporte.

Organización de Estados Iberoamericanos para la Educación, la Ciencia y la Cultura, OEl. (2013). Metas educativas para el 2021. Madrid: OEI.

Prats, E. (2013). Jugando a ser dioses: puntos críticos de las tecnologías en la educación. Revista Teoría de la Educación: Educación y Cultura en la Sociedad de la Información (TESI), 14 (3), 101-119.

Real, J., Cascales, A. \& Marcos, B. (2012). Uso compartido de pizarras digitales según los estilos de aprendizaje. [V Congreso Mundial de Estilos de Aprendizaje], Santander, 27, 28 y 29 de junio de 2012. 
Toledo, P. \& Sánchez, J.M. (2013). Situación actual de las pizarras digitales interactivas en las aulas de primaria. Revista de Educación a Distancia (RED), 43, 1-18.

Torras-Virgili, M.E. (2013). El aprendizaje colaborativo en línea y la ética del cuidado. Estudios sobre educación (ESE), 24, 149-171.

Tosato, P., Carramolino, B., \& Rubia Avi, B. (2014). Sharing Resources in Open Educational Communities. Qualitative Research in Education, 3 (1), 206-231.

UNESCO (2008). Estándares de competencia en TIC para docentes. Eduteka. 